\title{
ECCEMA ALÉRGICO DE CONTACTO PALPEBRAL POR KETOROLACO TROMETAMOL 0,5\% TÓPICO
}

\section{EYELID CONTACT ALLERGIC ECZEMA CAUSED BY TOPICAL KETOROLAC TROMETHAMINE 0.5\%}

\author{
RODRÍGUEZ NA ${ }^{1}$, ABARZUZA R ${ }^{1}$, CRISTÓBAL JA ${ }^{2}$, SIERRA ${ }^{1}$, MÍNGUEZ E ${ }^{1}$, DEL BUEY MA ${ }^{1}$
}

\begin{abstract}
RESUMEN
Caso clínico: Hombre de 65 años que había sido tratado con AINE tópico (Ketorolaco). El paciente presentó inyección conjuntival, edema de párpados, y dermatitis periorbitaria por eccema alérgico de contacto secundario a AINE, un hecho raro en este tipo de tratamiento.

Discusión: Este es un caso raro, ya que la aplicación de Ketorolaco tópico es seguro en la mayoría de los pacientes oftalmológicos. Además, las reacciones adversas asociadas con el Ketorolaco son similares a otros AINES.
\end{abstract}

Palabras clave: Alergia, eccema de contacto, Ketorolaco, asma, edema, dermatitis.

\begin{abstract}
Clinical case: A 65-year-old man had been treated with eye-drops containing NSAIDs (Ketorolac). He developed conjunctival injection, edematous swelling of the eyelids and periorbital dermatitis due to a contact allergy. Allergy to NSAIDs is uncommon. Discussion: This is an unusual case because topical application of ketorolac is safe in the vast majority of ophthalmologic patients. However adverse events associated with Ketorolac are similar to that of other NSAIDs (Arch Soc Esp Oftalmol 2006; 81: 213-216).
\end{abstract}

Key words: Allergic, contact eczema, Ketorolaco, asthma, oedema, dermatitis.

\section{INTRODUCCIÓN}

El uso de antiinflamatorios no esteroideos (AINE) tópicos en oftalmología, está ampliamente extendido para gran variedad de patologías como conjuntivitis alérgica, conjuntivitis vernal, inflamación de segmento anterior, edema macular quístico, prevención de la miosis quirúrgica, tratamiento de la inflamación ocular postoperatoria y tras cirugía refractiva, con el fin de reducir los procesos inflamatorios mediados por prostaglandinas.

El eccema alérgico es una intolerancia cutánea frente a diferentes agentes exógenos y endógenos, que desencadenan una reacción de hipersensibili-

Recibido: 19/10/05. Aceptado: 17/4/06.

Servicio de Oftalmología. Hospital Clínico Universitario Lozano Blesa. Zaragoza. España.

1 Licenciado en Medicina.

2 Doctor en Medicina. Jefe de Servicio.

Correspondencia:

Nelson Arturo Rodríguez Marco

Hospital Clínico Universitario Lozano Blesa

C/. San Juan Bosco, 15

50009 Zaragoza

España

E-mail: Nelson5000@latinmail.com 
dad tipo IV (mediada por células o retardada). Origina una dermoepidermitis superficial en la que coexisten varias lesiones elementales: eritema, vesícula, erosión, exudación, costra, descamación y liquenificación (1).

Los preparados tópicos y cosméticos son los principales causantes de eccemas alérgicos de contacto en los párpados. Los signos clínicos incluyen, inyección conjuntival, blefaritis, dermatitis periorbitaria y edema palpebral, acompañado de prurito (2).

El Ketorolaco trometamol 0,5\% es un AINE tópico de segunda generación del grupo pirrolo-pirrol, es una sal blanca en solución acuosa, y actúa por inhibición de la enzima ciclooxigenasa, esencial para la biosíntesis de prostaglandinas.

\section{CASO CLÍNICO}

Varón de 61 años que acudió a urgencias con lesiones eritemato-descamativas en párpados superiores e inferiores, supuración, formación de costras cutáneas y prurito, lo que se etiquetó de eccema alérgico de contacto en fase exudativo costrosa (fig. 1). No refería disminución de la agudeza visual. A la exploración presentaba hiperemia conjuntival grado II-III, cornea fluoresceína negativa, reacción folículo-papilar intensa y secreción serosa. Cataratas corticonucleares grado II-III, que iban a ser intervenidas. La presión intraocular y el estudio de fondo de ojo eran normales.

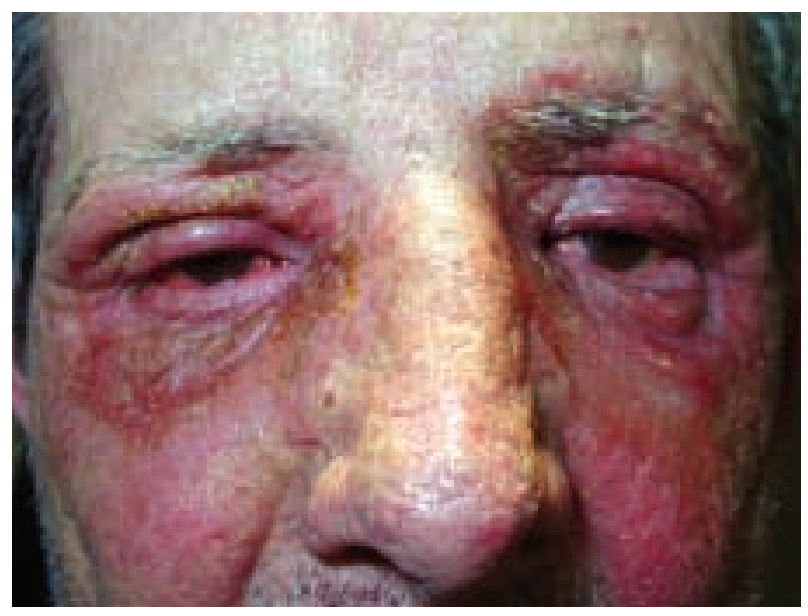

Fig. 1: Eccema alérgico de contacto que afecta a los cuatro párpados. Lesiones eritemato-descamativas de los párpados superiores e inferiores, supuración y formación de costras cutáneas.
Como antecedente, el paciente estaba siendo tratado profilácticamente a la intervención de cataratas aplicándose hacía cuatro días colirio de Ketorolaco trometamol (Acular ${ }^{\mathrm{TM}}$, Allergan SA, Tres Cantos, Madrid, España) y norfloxacino (Chibroxin, Merck Sharp \& Dohme de España, S.A.; Madrid, España) tres veces al día.

Ante esta situación se suprimieron los colirios, y se administraron $60 \mathrm{mg}$ de metilprednisolona intramuscular en urgencias y pomada de acetónido de fluocinolona tres veces al día, durante quince días. Tras dos semanas, el paciente mejoró de sus lesiones (figs. 2 y 3 ).

El paciente fue remitido al servicio de Alergología, para la realización de pruebas epicutáneas de provocación, «True test», correspondientes a Ketorolaco, Norfloxacino y Fenilefrina. El «True test» realizado con solución de colirio sin diluir y lectura a las 48, 72 y 120 horas, resultó positiva para Ketorolaco y Fenilefrina (fig. 4).

\section{DISCUSIÓN}

El uso de AINES tópicos está ampliamente extendido en oftalmología para el tratamiento de diferentes procesos de base inflamatoria como, conjuntivitis alérgica, conjuntivitis vernal, lesiones corneales, edema macular quístico, en el preoperatorio y postoperatorio de la cirugía ocular, etc. No obstante existen múltiples reacciones adversas asociadas a su uso tópico como ardor, disminución de la sensibilidad corneal, queratopatía, ulceraciones, adelgazamiento corneal y escleral e incluso perforación corneal (3).

El eccema es un proceso dinámico que pasa por diferentes etapas: un estadio agudo y un estadio crónico. Dentro del estadio agudo diferenciamos tres fases, fase de eritema y edema, fase de vesiculización, y fase de exudación, en las que las vesículas se rompen dejando pequeñas erosiones de las que fluye abundante exudado seroso que al secarse forma costras, y que es la fase en que se encontraba nuestro paciente (1).

En los ensayos clínicos con Ketorolaco las reacciones adversas más frecuentes son ojo rojo, picor y escozor transitorio, y otros síntomas menores de irritación ocular, tales como hiperemia, hinchazón bajo el ojo y leve edema palpebral. De acuerdo con estos criterios, una revisión que incluyó 1.055 individuos que recibieron Ketorolaco en los estudios de 

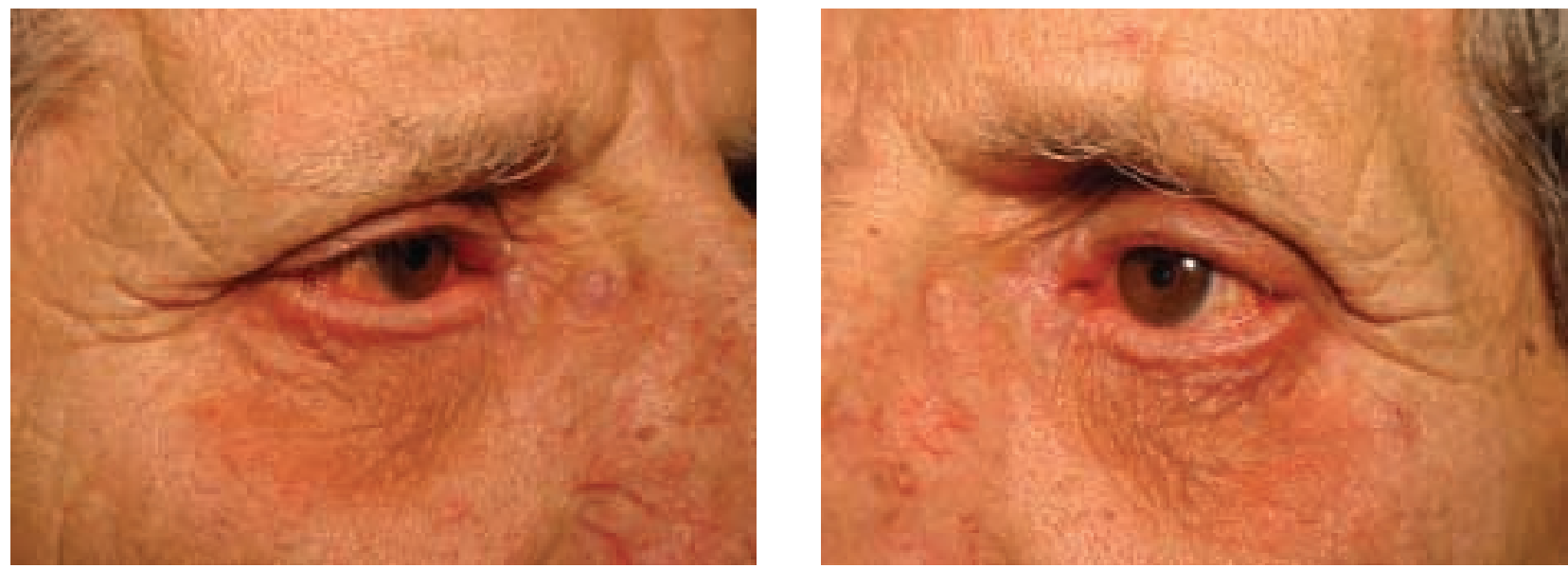

Fig. 2 y 3: Aspecto de los párpados tras dos semanas de tratamiento con acetónido de fluocinolona (Gelidina,) tres veces al día durante catorce días. Desaparición de las lesiones exudativo costrosas.

farmacología clínica y en ensayos clínicos, se mostró que sólo 32 (3\%) habían tenido una reacción alérgica. Con estos mismos criterios, 20 de 801 $(2,5 \%)$ de los pacientes tratados con el vehículo habían tenido una reacción alérgica (Datos de archivo, Allergan, Inc. Syntex study report CL5654, Ophthalmology clinical research department. Ketorolac tromethamine ophthalmic solution $0,5 \%$ : integrated summary of safety information. 1992). No obstante en la literatura se han descrito casos de

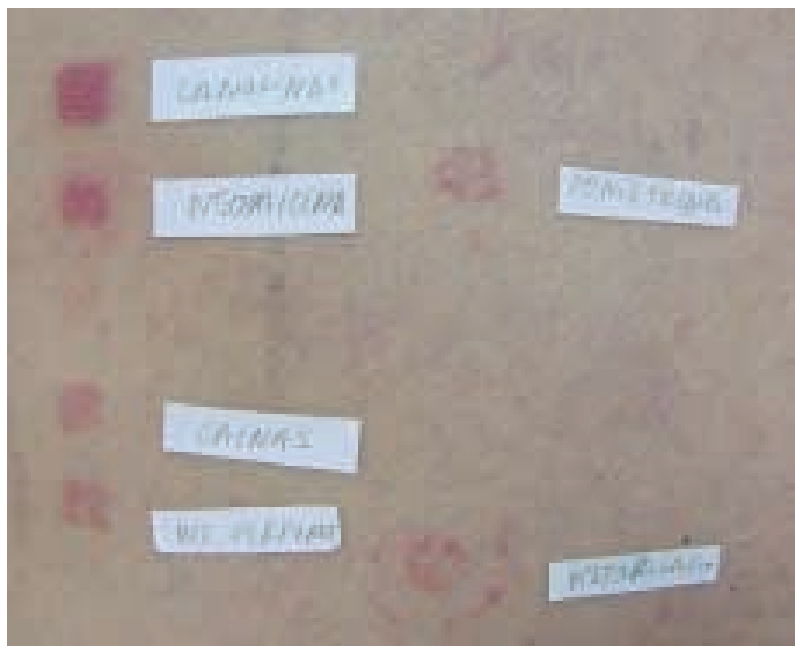

Fig. 4: Pruebas epicutáneas de provocación, «True test», para Ketorolaco, Norfloxacino y Fenilefrina. El «True test» con solución de colirio sin diluir resultó positiva para Ketorolaco (abajo derecha) y Fenilefrina. Mostró sensibilidad a alcoholes de lana, mezcla de caínas y neomocina. agudización de asma y broncoespasmo, tras la administración tópica de Ketorolaco, en pacientes alérgicos a AINES, o en aquellos que asociaban intolerancia a la aspirina, asma y pólipos nasales (Triada ASA) (4). De ahí que se deba tener precaución cuando se administre Ketorolaco a pacientes con sensibilidad conocida al ácido acetilsalicílico, derivados del ácido fenilacético, u otros AINE porque puede ocurrir sensibilidad cruzada.

Dentro de las quinolonas los efectos adversos, cuando se administran por vía sistémica son gastrointestinales, artropatía en pacientes jóvenes, nefritis intersticial, hematuria, fallo renal, etc. Un hecho destacable de las quinolonas, es la fototoxicidad, que parece ser el mecanismo de acción por el que aparece fotosensibilidad cutánea, principalmente en ancianos con enfermedades concomitantes que hallan precisado tratamiento antibiótico previo prolongado. En la literatura médica también hemos encontrado un solo caso de edema de párpado y enrojecimiento ocular leve tras la administración tópica de ciprofloxacino, al estudiar 31 casos de reacciones adversas a las quinolonas.

El caso que presentamos es el primero del que tenemos constancia de un eccema alérgico de contacto bilateral afectando a los cuatro párpados tras la administración tópica de Ketorolaco trometamol $0,5 \%$.

\section{BIBLIOGRAFÍA}

1. Dermatitis eccematosa. In: Fitzpatrick TB, Johnson RA, Polano M, Suurmond D, Wolf K. Atlas de Dermatología 
Clínica. III edición. México DF: Interamericana McGraw-Hill; 1998; 48-75.

2. Blum A, Brummer C, Lischka G. Edematous swelling of the eyelids caused by contact allergy. Hautarzt 1998; 49: 651-653.

3. Aragona P, Tripodi $G$, Spinella $R$, Lagana E, Ferreri $G$.
The effects of the topical administration of non-steroidal anti-inflamatory drugs on corneal epithelium and corneal sensitivity in normal subjets. Eye 2000; 14: 206-210.

4. Sitenga GL, Ing EB, Van Dellen RG, Younge BR, Leavitt JA. Asthma caused by topical application of ketorolac. Ophthalmology 1996; 103: 890-892. 